\title{
Abelian Repetitions in Sturmian Words
}

\author{
Gabriele Fici ${ }^{1}$, Alessio Langiu ${ }^{2}$, Thierry Lecroq ${ }^{3}$, Arnaud Lefebvre ${ }^{3}$, \\ Filippo Mignosi ${ }^{4}$ and Élise Prieur-Gaston ${ }^{3}$ \\ 1 Dipartimento di Matematica e Informatica, Università di Palermo, Italy \\ Gabriele.Fici@unipa.it \\ 2 Department of Informatics, King's College London, London, UK \\ Alessio.Langiu@kcl.ac.uk \\ 3 Normandie Université, LITIS EA4108, Université de Rouen, 76821 \\ Mont-Saint-Aignan Cedex, France \\ \{Thierry.Lecroq, Arnaud.Lefebvre, Elise.Prieur\}@univ-rouen.fr \\ 4 Dipartimento di Informatica, Università dell'Aquila, L'Aquila, Italy \\ Filippo.Mignosi@di.univaq.it
}

\begin{abstract}
We investigate abelian repetitions in Sturmian words. We exploit a bijection between factors of Sturmian words and subintervals of the unitary segment that allows us to study the periods of abelian repetitions by using classical results of elementary Number Theory. If $k_{m}$ denotes the maximal exponent of an abelian repetition of period $m$, we prove that $\lim \sup k_{m} / m \geq \sqrt{5}$ for any Sturmian word, and the equality holds for the Fibonacci infinite word. We further prove that the longest prefix of the Fibonacci infinite word that is an abelian repetition of period $F_{j}, j>1$, has length $F_{j}\left(F_{j+1}+F_{j-1}+1\right)-2$ if $j$ is even or $F_{j}\left(F_{j+1}+F_{j-1}\right)-2$ if $j$ is odd. This allows us to give an exact formula for the smallest abelian periods of the Fibonacci finite words. More precisely, we prove that for $j \geq 3$, the Fibonacci word $f_{j}$ has abelian period equal to $F_{n}$, where $n=\lfloor j / 2\rfloor$ if $j=0,1,2 \bmod 4$, or $n=1+\lfloor j / 2\rfloor$ if $j=3$ $\bmod 4$.
\end{abstract}

\section{Introduction}

The study of repetitions in words is a classical subject in Theoretical Computer Science both from the combinatorial and the algorithmic point of view. Repetitions are strictly related to the notion of periodicity. Recall that a word $w$ of length $|w|$ has a period $p>0$ if $w[i]=w[i+p]$ for any $1 \leqslant i \leqslant|w|-p$, where $w[i]$ is the symbol in position $i$ of $w$. Every word $w$ has a minimal period $p \leq|w|$. If $|w| / p \geq 1$, then $w$ is called a repetition of period $p$ and exponent $|w| / p$. When $|w| / p=k$ is an integer, the word $w$ is called an integer power, since it can be written as $w=u^{k}$, i.e., $w$ is the concatenation of $k$ copies of a word $u$ of length $p$. If instead $|w| / p$ is not an integer, the word $w$ is called a fractional power. So one can write $w=u^{k} v$, where $v$ is the prefix of $u$ such that $|w| / p=k+|v| /|u|$. For example, the word $w=a a b a a b a$ is a $7 / 3$-power since it has minimal period 3 and length 7. A classical reference on periodicity is [1, Chap. 7]. 
Abelian properties concerning words have been studied since the very beginning of Formal Languages and Combinatorics on Words. The notion of Parikh vector has become a standard and is often used without an explicit reference to the original 1966 Parikh's paper 2. Abelian powers were first considered in 1961 by Erdös [3] as a natural generalization of usual powers. Research concerning abelian properties of words and languages developed afterwards in different directions. In particular, there is a recent increasing of interest on abelian properties of words linked to periodicity (see, for example, [4/5|6|7|89]), and on the algorithmic search of abelian periodicities in strings [10|11|12 13 14].

Recall that the Parikh vector $\mathcal{P}_{w}$ of a finite word $w$ enumerates the cardinality of each letter of the alphabet in $w$. Therefore, two words have the same Parikh vector if one can be obtained from the other by permuting letters. We say that the word $w$ is an abelian repetition of (abelian) period $m$ and exponent $|w| / m$ if $w$ can be written as $w=u_{0} u_{1} \cdots u_{j-1} u_{j}$ for words $u_{i}$ and an integer $j>2$, where for $0<i<j$ all the $u_{i}$ 's have the same Parikh vector $\mathcal{P}$ whose sum of components is $m$ and the Parikh vectors of $u_{0}$ and $u_{j}$ are contained in $\mathcal{P}$ (see [15]). When $u_{0}$ and $u_{j}$ are empty, $w$ is called an abelian power or weak repetition [16. For example, the word $w=a b a a b$ is an abelian repetition of period 2 , since one can set $u_{0}=a, u_{1}=b a, u_{2}=a b$ and $u_{3}=\varepsilon$, where $\varepsilon$ denotes the empty word.

It is well known that Sturmian words and Fibonacci words, in particular, are extremal cases for several problems related to repetitions (see for example [17/18 19]) and are worst-case examples for classical pattern matching algorithms, e.g. Knuth-Morris-Pratt [20/21]. There exists a huge bibliography concerning Sturmian words (see for instance the survey papers [22 23, [1, Chap. 2], [24, Chap. 6] and references therein). In particular, there is an analogous result to the one presented in this paper concerning classical repetitions in the Fibonacci infinite word 25. In 26], a bijection between factors of Sturmian words and subintervals of the unitary segment is described. We show in this paper that this bijection preserves abelian properties of factors (see Proposition 4). Therefore, we are able to apply techniques of Number Theory coupled with Combinatorics on Words to obtain our main results. More precisely, if $k_{m}$ denotes the maximal exponent of an abelian repetition of period $m$, we prove that $\lim \sup k_{m} / m \geq \sqrt{5}$ for any Sturmian word, and the equality holds for the Fibonacci infinite word.

We further prove that for any Fibonacci number $F_{j}, j>1$, the longest prefix of the Fibonacci infinite word that is an abelian repetition of period $F_{j}$ has length $F_{j}\left(F_{j+1}+F_{j-1}+1\right)-2$ if $j$ is even or $F_{j}\left(F_{j+1}+F_{j-1}\right)-2$ if $j$ is odd (Theorem 7). This allows us to give an exact formula for the smallest abelian periods of the Fibonacci finite words. More precisely, we prove, in Theorem 8 , that for $j \geq 3$, the Fibonacci word $f_{j}$ has abelian period equal to $F_{n}$, where $n=\lfloor j / 2\rfloor$ if $j=0,1,2 \bmod 4$, or $n=1+\lfloor j / 2\rfloor$ if $j=3 \bmod 4$.

Due to space constraints the proofs are omitted, but they will be included in an upcoming full version of the paper. 


\section{Preliminaries}

Let $\Sigma=\left\{a_{1}, a_{2}, \ldots, a_{\sigma}\right\}$ be a finite ordered alphabet of cardinality $\sigma$ and $\Sigma^{*}$ the set of words over $\Sigma$. We denote by $|w|$ the length of the word $w$. We write $w[i]$ the $i$-th symbol of $w$ and $w[i . . j]$ the factor of $w$ from the $i$-th symbol to the $j$-th symbol, with $1 \leqslant i \leqslant j \leqslant|w|$. We denote by $|w|_{a}$ the number of occurrences of the symbol $a \in \Sigma$ in the word $w$.

The Parikh vector of a word $w$, denoted by $\mathcal{P}_{w}$, counts the occurrences of each letter of $\Sigma$ in $w$, i.e., $\mathcal{P}_{w}=\left(|w|_{a_{1}}, \ldots,|w|_{a_{\sigma}}\right)$. Given the Parikh vector $\mathcal{P}_{w}$ of a word $w$, we denote by $\mathcal{P}_{w}[i]$ its $i$-th component and by $\left|\mathcal{P}_{w}\right|$ the sum of its components. Thus, for a word $w$ and $1 \leqslant i \leqslant \sigma$, we have $\mathcal{P}_{w}[i]=|w|_{a_{i}}$ and $\left|\mathcal{P}_{w}\right|=\sum_{i=1}^{\sigma} \mathcal{P}_{w}[i]=|w|$. Finally, given two Parikh vectors $\mathcal{P}, \mathcal{Q}$, we write $\mathcal{P} \subset \mathcal{Q}$ if $\mathcal{P}[i] \leqslant \mathcal{Q}[i]$ for every $1 \leqslant i \leqslant \sigma$ and $|\mathcal{P}|<|\mathcal{Q}|$.

Following [15], we give the definition below.

Definition 1. A word $w$ is an abelian repetition of period $m>0$ and exponent $|w| / m=k$ if one can write $w=u_{0} u_{1} \cdots u_{j-1} u_{j}$ for some $j>2$ such that $\mathcal{P}_{u_{0}} \subset \mathcal{P}_{u_{1}}=\ldots=\mathcal{P}_{u_{j-1}} \supset \mathcal{P}_{u_{j}}$, and $\left|\mathcal{P}_{u_{1}}\right|=\ldots=\left|\mathcal{P}_{u_{j-1}}\right|=m$.

An abelian power is an abelian repetition in which $u_{0}=u_{j}=\varepsilon$.

We call $u_{0}$ and $u_{j}$ the head and the tail of the abelian repetition, respectively. Notice that the length $t=\left|u_{j}\right|$ of the tail is uniquely determined by $h=\left|u_{0}\right|, m$ and $|w|$, namely $t=(|w|-h) \bmod m$.

Example 1. The word $w=$ abaababa is an abelian repetition of period 2 and exponent 4 , since one can write $w=a \cdot b a \cdot a b \cdot a b \cdot a$. Notice that $w$ is also an abelian repetition of period 3 and exponent $8 / 3$, since $w=\varepsilon \cdot a b a \cdot a b a \cdot b a$.

In the rest of the paper, when we refer to an abelian repetition of period $m$, we always suppose that $m$ is the minimal abelian period of $w$.

Remark 1. We adopt the convention that an abelian repetition of exponent $k \geq 2$ has also exponent $k^{\prime}$ for any real number $k^{\prime}$ such that $2 \leq k^{\prime} \leq k$. This is a standard convention widely adopted in the classical case.

\subsection{Sturmian words}

From now on, we fix the alphabet $\Sigma=\{\mathbf{a}, \mathbf{b}\}$. We start by recalling a bijection between factors of Sturmian words and subintervals of the unitary segment introduced in [26].

Let $\alpha$ and $\rho$ be two real numbers with $\alpha \in(0,1)$. Following the notations of [27, the fractional part of a number $r$ is defined by $\{r\}=r-\lfloor r\rfloor$, where $\lfloor r\rfloor$ is the greatest integer smaller than or equal to $r$. Therefore, for $\alpha \in(0,1)$, one has that $\{-\alpha\}=1-\alpha$.

The sequence $\{n \alpha+\rho\}, n>0$, defines an infinite word $s_{\alpha, \rho}=a_{1}(\alpha, \rho) a_{2}(\alpha, \rho) \cdots$ by the rule

$$
a_{n}(\alpha, \rho)=\left\{\begin{array}{l}
\mathbf{b} \text { if }\{n \alpha+\rho\} \in[0,\{-\alpha\}) \\
\mathbf{a} \text { if }\{n \alpha+\rho\} \in[\{-\alpha\}, 1) .
\end{array}\right.
$$


See Fig. 1 for a graphical illustration.

We will write $a_{n}$ instead of $a_{n}(\alpha, \rho)$ whenever there is no possibility of mistake. If $\alpha$ is rational, i.e. $\alpha=n / m$, with $n$ and $m$ coprime integers, then it is easy to prove that the word $s_{\alpha, \rho}$ is periodic and $m$ is its minimal period. In this case, $s_{\alpha, \rho}$ is also periodic in the abelian sense, since it trivially has abelian period $m$.

If instead $\alpha$ is irrational, then $s_{\alpha, \rho}$ is not periodic and is called a Sturmian word. Therefore, in the rest of the paper, we always suppose $\alpha$ irrational.

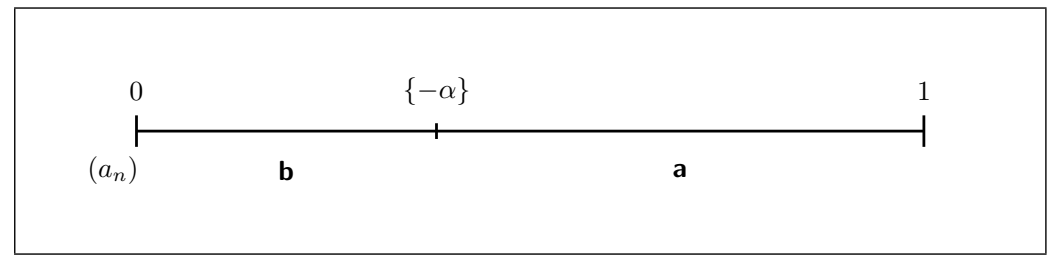

Fig. 1. An application of Proposition 1 when $\alpha=\phi-1 \approx 0.618$ (thus $\{-\alpha\} \approx 0.382$ ) for $i=0$. If $\{n \alpha+\rho\} \in[\{-\alpha\}, 1)$, then $a_{n}=\mathbf{a}$; otherwise $a_{n}=\mathbf{b}$.

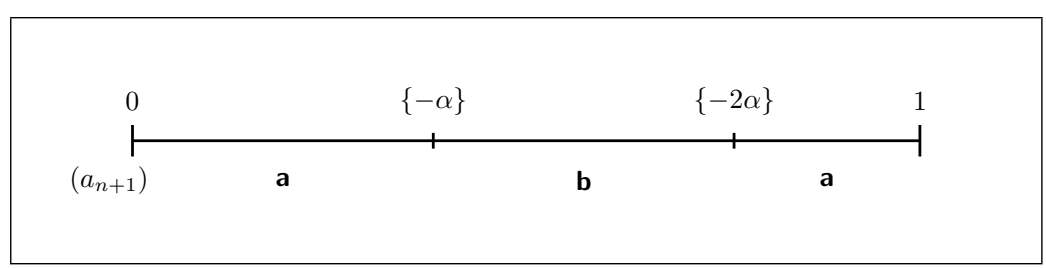

Fig. 2. An application of Proposition 1 when $\alpha=\phi-1 \approx 0.618$ (thus $\{-\alpha\} \approx 0.382$ ) for $i=1$. If $\{n \alpha+\rho\} \in[0,\{-\alpha\}) \cup[\{-2 \alpha\}, 1)$, then $a_{n+1}=\mathbf{a}$; otherwise $a_{n+1}=\mathbf{b}$.

\begin{tabular}{|ccccc|}
\hline & $\{-\alpha\}$ & & \\
$\left(a_{n}\right)$ & & $\{-2 \alpha\}$ & \\
$\left(a_{n+1}\right)$ & $\mathbf{b}$ & & $\mathbf{a}$ & \\
& & $\mathbf{b}$ & $\mathbf{a}$ & \\
& & & \\
\end{tabular}

Fig. 3. A single graphic representation of the information given in Fig. 1 and 2 If $\{n \alpha+\rho\} \in[0,\{-\alpha\})=L_{0}(\alpha, 2)$, then $a_{n}=\mathbf{b}, a_{n+1}=\mathbf{a}$. If $\{n \alpha+\rho\} \in[\{-\alpha\},\{-2 \alpha\})=$ $L_{1}(\alpha, 2)$, then $a_{n}=\mathbf{a}, a_{n+1}=\mathbf{b}$. If $\{n \alpha+\rho\} \in[\{-2 \alpha\}, 1)=L_{2}(\alpha, 2)$, then $a_{n}=\mathbf{a}$, $a_{n+1}=\mathbf{a}$. 
Example 2. For $\alpha=\phi-1$ and $\rho=0$, where $\phi=(1+\sqrt{5}) / 2$ is the golden ratio, one obtains the Fibonacci infinite word

$$
f=\text { abaababaabaababaababa } \ldots
$$

Remark 2. Since $\alpha \in(0,1)$, we have $\{-i \alpha\} \neq\{-(i+1) \alpha\}$ for any natural number $i$. We shall use this fact freely and with no explicit mention.

It is possible to prove (see [26, Corollary 2.3]) that the following result holds.

Proposition 1. Let $\alpha$ and $\rho$ be real numbers, with $\alpha \in(0,1)$ irrational. For any natural numbers $n$, $i$, with $n>0$, if $\{-(i+1) \alpha\}<\{-i \alpha\}$ then

$$
a_{n+i}=\mathbf{a} \Longleftrightarrow\{n \alpha+\rho\} \in[\{-(i+1) \alpha\},\{-i \alpha\}),
$$

whereas if $\{-i \alpha\}<\{-(i+1) \alpha\})$ then

$$
a_{n+i}=\mathbf{a} \Longleftrightarrow\{n \alpha+\rho\} \in[0,\{-i \alpha\}) \cup[\{-(i+1) \alpha\}, 1) .
$$

In Fig. 1 and 2 we display a graphical representation of the formula given in Proposition 1 for $\alpha=\phi-1$ when $i=0$ and $i=1$, respectively. In Fig. 3 we present within a single graphic the situations illustrated in Fig. 1 and 2 ,

Let $m$ be a positive integer. Consider the $m+2$ points $0,1,\{-i \alpha\}$, for $1 \leq$ $i \leq m$. Rearranging these points in increasing order one has:

$$
0=c_{0}(\alpha, m)<c_{1}(\alpha, m)<\ldots<c_{k}(\alpha, m)<\ldots<c_{m}(\alpha, m)<c_{m+1}(\alpha, m)=1 .
$$

One can therefore define the $m+1$ non-empty subintervals

$$
L_{k}(\alpha, m)=\left[c_{k}(\alpha, m), c_{k+1}(\alpha, m)\right), 0 \leq k \leq m .
$$

By using Proposition 1, it is possible to associate with each interval $L_{k}(\alpha, m)$ a factor of length $m$ of the word $s_{\alpha, \rho}$, and this correspondence is bijective (see [28]). We call this correspondence the Sturmian bijection.

Proposition 2. Each factor of $s_{\alpha, \rho}$ of length $m, a_{n} a_{n+1} \cdots a_{n+m-1}$, depends only on the interval $L_{k}(\alpha, m)$ containing the point $\{n \alpha+\rho\}$; more precisely, it depends only on the set $I_{k}(\alpha, m)$ of integers $i \in\{0,1, \ldots, m-1\}$ such that either $\{-(i+1) \alpha\}<\{-i \alpha\}$ and $c_{k}(\alpha, m) \in[\{-(i+1) \alpha\},\{-i \alpha\})$ or $\{-(i+1) \alpha\}>$ $\{-i \alpha\}$ and $c_{k}(\alpha, m) \notin[\{-i \alpha\},\{-(i+1) \alpha\})$. The set $I_{k}(\alpha, m)$ is the set of the integers $i$, with $0 \leq i \leq m-1$, such that $a_{n+i}=\mathbf{a}$.

Corollary 1. Since the set of factors of $s_{\alpha, \rho}$ depends only on the sequence $\{-i \alpha\}, i>0$, it does not depend on $\rho$. In particular, then, for any $\rho$ the word $s_{\alpha, \rho}$ has the same set of factors of the word $s_{\alpha, 0}$.

Example 3. Let $\alpha=\phi-1$. In Fig. 3 we show an example of the Sturmian bijection when $m=2$. The ordered sequence of points defining the subintervals $L_{k}(\alpha, 2)$ is

$c_{0}(\alpha, 2)=0, c_{1}(\alpha, 2)=\{-\alpha\} \approx 0.382, c_{2}(\alpha, 2)=\{-2 \alpha\} \approx 0.764, c_{3}(\alpha, 2)=1$. 


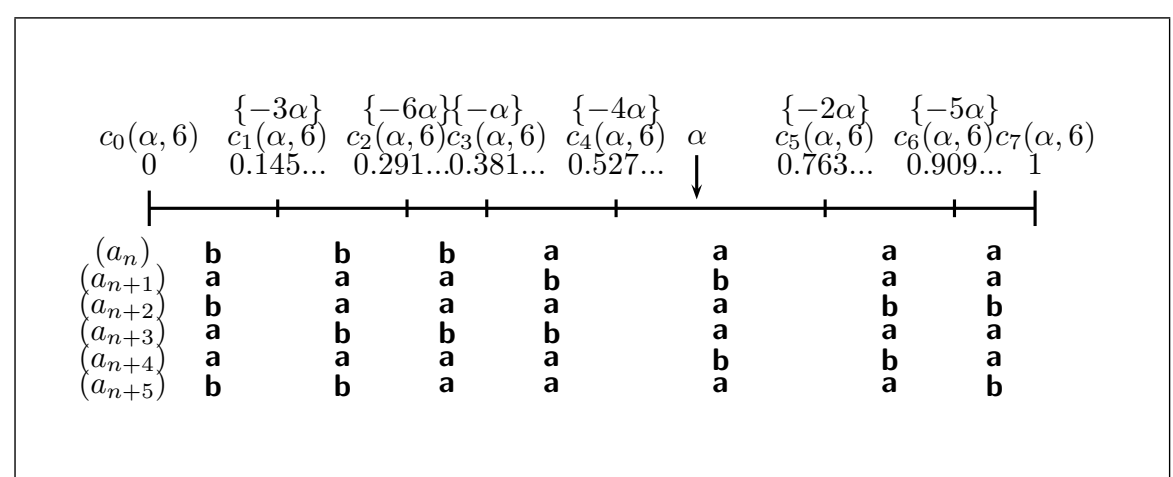

Fig. 4. The subintervals $L_{k}(\alpha, m)$ of the Sturmian bijection obtained for $\alpha=\phi-1$ and $m=6$. Below each interval there is the factor of $s_{\alpha}$ of length 6 associated with that interval. For $\rho=0$ and $n=1$, the prefix of length 6 of the Fibonacci word is associated with $L_{4}(\alpha, 6)=\left[c_{4}(\alpha, 6), c_{5}(\alpha, 6)\right)$, which is the interval containing $\alpha$.

In Fig. 4 we show an example of the Sturmian bijection when $\alpha=\phi-1$ and $m=6$. Below each interval there is the factor of $s_{\alpha}$ of length $m=6$ associated with that interval. The prefix of length 6 of the Fibonacci word corresponds to the factor below the interval containing $\alpha$ (so, for $n=1$ and $\rho=0$ ). Notice that all the factors of length 6 of the Fibonacci word appear, and moreover they are lexicographically ordered from right to left. This property concerning lexicographic order holds for any Sturmian word and any length $m$ of factors, and is stated in next proposition, which is of independent interest and is related to some recent research on Sturmian words and the lexicographic order (see 29130/31/32]).

Proposition 3. Let $m \geq 1$ and $k, k^{\prime}$ such that $0 \leq k, k^{\prime} \leq m$. Then $k<k^{\prime}$ if and only if the factor $t_{\alpha, \rho, m}$ associated to $L_{k}(\alpha, m)$ in the Sturmian bijection is lexicographically greater than the factor $t_{\alpha, \rho, m}^{\prime}$ associated to $L_{k^{\prime}}(\alpha, m)$.

In the next section we present a new property of the Sturmian bijection, that will allow us to use some standard Number Theory techniques to deal with abelian repetitions in Sturmian words and, in particular, in the Fibonacci infinite word. Similar techniques are used in $[8]$ to derive some other interesting results on abelian powers in Sturmian words.

\section{Sturmian bijection and Parikh vectors}

Let $s_{\alpha, \rho}$ be a Sturmian word. Since we are mainly interested in the set of factors of $s_{\alpha, \rho}$, we do not lose generality, by Corollary 1 , supposing $\rho=0$. The Sturmian words with $\rho=0$ are called characteristic, and have been the object of deep studies within the field of Sturmian words. For simplicity of notation, we will write $s_{\alpha}$ instead of $s_{\alpha, 0}$. 
We now describe some properties of the Sturmian bijection between the factors of length $m$ of $s_{\alpha}$ and the subintervals $L_{k}(\alpha, m)$, that we will use to prove the main results of the paper.

Proposition 4. Under the Sturmian bijection, all the factors corresponding to an interval $c_{k}(\alpha, m)=[x, y)$ with $x \geq\{-m \alpha\}$ have the same Parikh vector $v_{1}(\alpha, m)$ and all the factors corresponding to an interval $[x, y)$ with $y \leq$ $\{-m \alpha\}$ have the same Parikh vector $v_{2}(\alpha, m)$. Moreover, one has $v_{1}(\alpha, m)[1]=$ $v_{2}(\alpha, m)[1]+1$.

The reader can see in Fig. 4 that the factors of length 6 corresponding to an interval to the left of $\{-6(\phi-1)\}$ have Parikh vector $(3,3)$, while the other ones have Parikh vector $(4,2)$.

We now address the following questions:

1. Given $m$, how large can be the exponent of an abelian repetition of period $m$ in $s_{\alpha}$ ?

2. What can we say in the particular case of the Fibonacci word, i.e., when $\alpha=\phi-1$ ?

The next result follows straightforwardly from Proposition 4

Corollary 2. Let $w$ be an abelian power of period $m$ and exponent $k+1$ appearing in $s_{\alpha}$ in position $n$. Then all the points in the sequence $\{n \alpha\},\{(n+$ $m) \alpha\},\{(n+2 m) \alpha\}, \ldots,\{(n+k m) \alpha\}$ are in the same subinterval in which $[0,1)$ is subdivided by the point $\{-m \alpha\}$, i.e., either $[0,\{-m \alpha\})$ or $[\{-m \alpha\}, 1)$.

The next proposition is a technical step to prove the following theorem.

Proposition 5. If $k \geq 1$, the $k+1$ points of Corollary 2 are naturally ordered. That is to say, if $\{m \alpha\}<0.5$, then they are all in the subinterval $[0,\{-m \alpha\})$ and one has $\{n \alpha\}<\{(n+m) \alpha\}<\ldots<\{(n+k m) \alpha\}$; if instead $\{m \alpha\}>0.5$ then they are all in the interval $[\{-m \alpha\}, 1)$ and one has $\{(n+k m) \alpha\}<\{(n+$ $(k-1) m) \alpha\}<\ldots<\{n \alpha\}$.

Theorem 1. Let $m$ be a positive integer such that $\{m \alpha\}<0.5$ (resp. $\{m \alpha\}>$ $0.5)$. Then:

1. In $s_{\alpha}$ there is an abelian power of period $m$ and exponent $k \geq 2$ if and only if $\{m \alpha\}<\frac{1}{k}$ (resp. $\left.\{-m \alpha\}<\frac{1}{k}\right)$.

2. If in $s_{\alpha}$ there is an abelian power of period $m$ and exponent $k \geq 2$ starting in position $i$ with $\{i \alpha\} \geq\{m \alpha\}$ (resp. $\{i \alpha\} \leq\{m \alpha\}$ ), then $\{m \alpha\}<\frac{1}{k+1}$ (resp. $\{-m \alpha\}<\frac{1}{k+1}$ ). Conversely, if $\{m \alpha\}<\frac{1}{k+1}$ (resp. $\{-m \alpha\}<\frac{1}{k+1}$ ), then there is an abelian power of period $m$ and exponent $k \geq 2$ starting in position $m$.

The previous theorem allows us to deal with abelian repetitions in a Sturmian word $s_{\alpha}$ by using classical results on the approximation of the irrational $\alpha$ by rationals. This is a classical topic in Number Theory. Since the number $\phi-1$ has special properties within this topic, we have in turn specific results for the Fibonacci infinite word. 


\section{Approximating irrationals by rationals and abelian repetitions}

We recall some classical results of Number Theory. For any notation not explicitly defined in this section we refer to [27, Chap. X, XI].

The sequence $F_{0}=1, F_{1}=1, F_{j+1}=F_{j}+F_{j-1}$ for $j \geq 1$ is the well known sequence of Fibonacci numbers. The sequence of fractions $\frac{F_{j+1}}{F_{j}}$ converges to $\phi=\frac{\sqrt{5}+1}{2}$, while the sequence $\frac{F_{j}}{F_{j+1}}$ converges to $\phi-1=\frac{\sqrt{5}-1}{2}$. Moreover, the sequences $\frac{F_{j+1}}{F_{j}}$ and $0=\frac{0}{1}, \frac{F_{j}}{F_{j+1}}, j=0,1, \ldots$, are the sequences of convergents, in the development in continued fractions, of $\phi$ and $\phi-1$ respectively.

Concerning the approximation given by the above convergents, the following result holds (see [27, Chap. X, Theorem 171] and [27, Chap. XI, Section 11.8]).

Theorem 2. For any $j>0$,

$$
\phi-\frac{F_{j+1}}{F_{j}}=(\phi-1)-\frac{F_{j-1}}{F_{j}}=\frac{(-1)^{j}}{F_{j}\left(\phi F_{j}+F_{j-1}\right)} .
$$

We also report the following theorems (see 27, Chap. XI, Theorem 193 and the proof of Theorem 194]).

Theorem 3. Any irrational $\alpha$ has an infinity of approximations which satisfy

$$
\left|\frac{n}{m}-\alpha\right|<\frac{1}{\sqrt{5} m^{2}}
$$

Theorem 4. Let $\alpha=\phi-1$. If $A>\sqrt{5}$, then the inequality

$$
\left|\frac{n}{m}-\alpha\right|<\frac{1}{A m^{2}}
$$

has only a finite number of solutions.

The last two theorems, coupled with the first part of Theorem 1 allow us to derive the next result.

Theorem 5. Let $s_{\alpha}$ be a Sturmian word. For any integer $m>1$, let $k_{m}$ be the maximal exponent of an abelian repetition of period $m$ in $s_{\alpha}$. Then

$$
\limsup _{m \rightarrow \infty} \frac{k_{m}}{m} \geq \sqrt{5},
$$

and the equality holds if $\alpha=\phi-1$. 


\section{Prefixes of the Fibonacci infinite word}

We now study the abelian repetitions that are prefixes of the Fibonacci infinite word. For this, we will make use of the second part of Theorem 1. Notice that an abelian repetition of period $m$ appearing as a prefix of the Fibonacci word can have a head of length equal to $m-1$ at most. Therefore, we have to check all the abelian powers that start in position $i$ for every $i=1, \ldots, m$. In order to do this, we report here another result (see [27, Chap. X, Theorem 182]).

Theorem 6. Let $n_{i} / m_{i}$ be the $i$-th convergent to $\alpha$. If $i>1,0<m \leq m_{i}$ and $n / m \neq n_{i} / m_{i}$, then $\left|n_{i}-m_{i} \alpha\right|<|n-m \alpha|$.

The previous theorem implies the following result.

Corollary 3. Suppose that $m>1$ is the denominator of a convergent to $\alpha$ and that $\{m \alpha\}<0.5$ (resp. $\{m \alpha\}>0.5$ ). Then for any $i$ such that $1 \leq i<m$, one has $\{i \alpha\} \geq\{m \alpha\}$ (resp. $\{i \alpha\} \leq\{m \alpha\}$ ).

From the previous corollary, we have that if $m>1$ is a Fibonacci number and $\alpha=\phi-1$, then the hypotheses of the second part of Theorem 1 are satisfied. The next proposition is a direct consequence of Corollary 3 . Theorem 1 and Theorem 2 .

Proposition 6. Let $j>1$. In the Fibonacci infinite word, the longest abelian power having period $F_{j}$ and starting in a position $i \leq F_{j}$ has an occurrence starting in position $F_{j}$, and has exponent equal to

$$
\left\lfloor\phi F_{j}+F_{j-1}\right\rfloor-1= \begin{cases}F_{j+1}+F_{j-1}-1 & \text { if } j \text { is even } ; \\ F_{j+1}+F_{j-1}-2 & \text { if } j \text { is odd } .\end{cases}
$$

The following theorem provides a formula for computing the length of the longest abelian repetition occurring as a prefix in the Fibonacci infinite word.

Theorem 7. Let $j>1$. The longest prefix of the Fibonacci infinite word that is an abelian repetition of period $F_{j}$ has length $F_{j}\left(F_{j+1}+F_{j-1}+1\right)-2$ if $j$ is even or $F_{j}\left(F_{j+1}+F_{j-1}\right)-2$ if $j$ is odd.

Corollary 4. Let $j>1$ and $k_{j}$ be the maximal exponent of a prefix of the Fibonacci word that is an abelian repetition of period $F_{j}$. Then

$$
\lim _{j \rightarrow \infty} \frac{k_{j}}{F_{j}}=\sqrt{5}
$$

In Fig. 5 we give a graphical representation of the longest prefix of the Fibonacci infinite word that is an abelian repetition of period $m$ for $m=2,3$ and 5 . In Table 1 we give the length $l p\left(F_{j}\right)$ of the longest prefix of the Fibonacci infinite word that is an abelian repetition of period $F_{j}$, for $j=2, \ldots, 11$, computed using the formula of Theorem 7 . We also show the values of the distance 


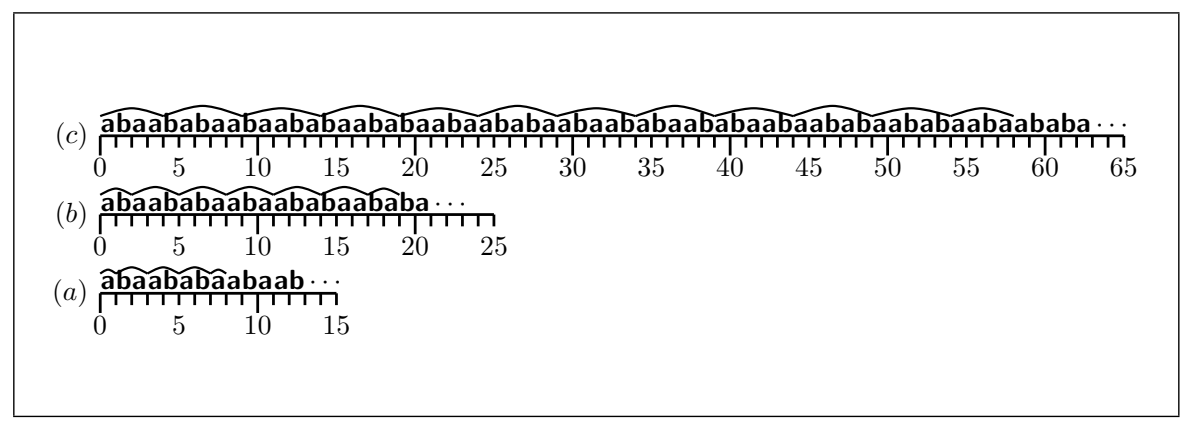

Fig. 5. Longest abelian repetition of period $m$ that is a prefix of the Fibonacci word for $m=2,3,5$. (a) For $m=2$, the longest abelian repetition has length $8=1+3 p+1$. (b) For $m=3$, the longest abelian repetition has length $19=2+5 p+2$. (c) For $m=5$, the longest abelian repetition has length $58=4+10 p+4$.

\begin{tabular}{ccccccccccc}
$j$ & 2 & 3 & 4 & 5 & 6 & 7 & 8 & 9 & 10 & 11 \\
\hline$F_{j}$ & 2 & 3 & 5 & 8 & 13 & 21 & 34 & 55 & 89 & 144 \\
\hline$l p\left(F_{j}\right)$ & 8 & 19 & 58 & 142 & 388 & 985 & 2616 & 6763 & 17798 & 46366 \\
\hline$\left|\sqrt{5}-k_{j} / F_{j}\right| \times 10^{2}$ & 23.6 & 12.5 & 8.393 & 1.732 & 5.98 & 0.25 & 2.69 & 0.037 & 1.087 & 0.005 \\
\hline
\end{tabular}

Table 1. The length of the longest prefix $\left(l p\left(F_{j}\right)\right)$ of the Fibonacci word having abelian period $F_{j}$ for $j=2, \ldots, 11$. The table also reports rounded distances (multiplied by $10^{2}$ ) between $\sqrt{5}$ and the ratio between the exponent $k_{j}=l p\left(F_{j}\right) / F_{j}$ of the longest prefix of the Fibonacci word having abelian period $F_{j}$ and $F_{j}$ (see Corollary 4 ).

between $\sqrt{5}$ and the ratio between the maximal exponent $k_{j}=l p\left(F_{j}\right) / F_{j}$ of a prefix of the Fibonacci infinite word having abelian period $F_{j}$ and $F_{j}$.

Recall that the Fibonacci (finite) words are defined by $f_{0}=\mathbf{b}, f_{1}=\mathbf{a}$, and for every $j>1, f_{j+1}=f_{j} f_{j-1}$. So, for every $j$, one has $\left|f_{j}\right|=F_{j}$. As a consequence of the formula given in Theorem 7 , we have the following result on the smallest abelian periods of the Fibonacci words.

Theorem 8. For $j \geq 3$, the (smallest) abelian period of the word $f_{j}$ is the $n$-th Fibonacci number $F_{n}$, where $n=\lfloor j / 2\rfloor$ if $j=0,1,2 \bmod 4$, or $n=1+\lfloor j / 2\rfloor$ if $j=3 \bmod 4$.

For example, the abelian period of the word $f_{4}=$ abaab is $2=F_{2}=\lfloor 4 / 2\rfloor$, since one can write $f_{4}=\mathbf{a} \cdot \mathbf{b a} \cdot \mathbf{a b}$; the abelian period of $f_{5}=$ abaababa is $2=F_{2}$; the abelian period of $f_{6}=$ abaababaabaab is $3=F_{3}$; the abelian period of $f_{7}=$ abaababaabaababaababa is $5=F_{4}$. In Table 2 we report the abelian periods of the first Fibonacci words.

We conclude the paper with the following open problems: 


\begin{tabular}{ccccccccccccccc}
$j$ & 3 & 4 & 5 & 6 & 7 & 8 & 9 & 10 & 11 & 12 & 13 & 14 & 15 & 16 \\
\hline a. p. of $f_{j}$ & $F_{2}$ & $F_{2}$ & $F_{2}$ & $F_{3}$ & $F_{4}$ & $F_{4}$ & $F_{4}$ & $F_{5}$ & $F_{6}$ & $F_{6}$ & $F_{6}$ & $F_{7}$ & $F_{8}$ & $F_{8}$ \\
\hline
\end{tabular}

Table 2. The (smallest) abelian periods of the Fibonacci words $f_{j}$ for $j=3, \ldots, 16$.

1. Is it possible to find the exact value of $\lim \sup \frac{k_{m}}{m}$ for other Sturmian words $s_{\alpha}$ with slope $\alpha$ different from $\phi-1$ ?

2. Is it possible to give the exact value of this superior limit when $\alpha$ is an algebraic number of degree 2 ?

\section{References}

1. Lothaire, M.: Algebraic Combinatorics on Words. Cambridge University Press, Cambridge, U.K. (2002)

2. Parikh, R.J.: On context-free languages. J. Assoc. Comput. Mach. 13 (1966) 570-581

3. Erdös, P.: Some unsolved problems. Magyar Tud. Akad. Mat. Kutato. Int. Kozl. 6 (1961) 221-254

4. Avgustinovich, S., Karhumäki, J., Puzynina, S.: On abelian versions of Critical Factorization Theorem. RAIRO Theor. Inform. Appl. 46 (2012) 3-15

5. Cassaigne, J., Richomme, G., Saari, K., Zamboni, L.: Avoiding Abelian powers in binary words with bounded Abelian complexity. Int. J. Found. Comput. Sci. 22 (2011) 905-920

6. Domaratzki, M., Rampersad, N.: Abelian primitive words. Int. J. Found. Comput. Sci. 23 (2012) 1021-1034

7. Puzynina, S., Zamboni, L.Q.: Abelian returns in Sturmian words. J. Comb. Theory, Ser. A 120 (2013) 390-408

8. Richomme, G., Saari, K., Zamboni, L.: Abelian complexity of minimal subshifts. Journal of the London Mathematical Society 83 (2011) 79-95

9. Samsonov, A., Shur, A.: On Abelian repetition threshold. RAIRO Theor. Inform. Appl. 46 (2012) 147-163

10. Christou, M., Crochemore, M., Iliopoulos, C.S.: Identifying all abelian periods of a string in quadratic time and relevant problems. Int. J. Found. Comput. Sci. 23 (2012) 1371-1384

11. Crochemore, M., Iliopoulos, C.S., Kociumaka, T., Kubica, M., Pachocki, J., Radoszewski, J., Rytter, W., Tyczynski, W., Walen, T.: A note on efficient computation of all abelian periods in a string. Inf. Process. Lett. 113 (2013) 74-77

12. Fici, G., Lecroq, T., Lefebvre, A., Prieur-Gaston, E.: Computing Abelian Periods in Words. In: Proceedings of the Prague Stringology Conference, PSC 2011, Czech Technical University in Prague (2011) 184-196

13. Fici, G., Lecroq, T., Lefebvre, A., Prieur-Gaston, E., Smyth, W.F.: Quasi-Linear Time Computation of the Abelian Periods of a Word. In: Proceedings of the Prague Stringology Conference, PSC 2012, Czech Technical University in Prague (2012) 103-110 
14. Kociumaka, T., Radoszewski, J., Rytter, W.: Fast algorithms for abelian periods in words and greatest common divisor queries. In: STACS 2013. Volume 20 of LIPIcs., Schloss Dagstuhl - Leibniz-Zentrum fuer Informatik (2013) 245-256

15. Constantinescu, S., Ilie, L.: Fine and Wilf's theorem for abelian periods. Bull. Eur. Assoc. Theoret. Comput. Sci. EATCS 89 (2006) 167-170

16. Cummings, L.J., Smyth, W.F.: Weak repetitions in strings. J. Combin. Math. Combin. Comput. 24 (1997) 33-48

17. Crochemore, M., Ilie, L., Rytter, W.: Repetitions in strings: Algorithms and combinatorics. Theoret. Comput. Sci. 410 (2009) 5227-5235

18. Iliopoulos, C.S., Moore, D., Smyth, W.F.: A Characterization of the Squares in a Fibonacci String. Theoret. Comput. Sci. 172 (1997) 281-291

19. Mignosi, F., Restivo, A.: Characteristic Sturmian words are extremal for the critical factorization theorem. Theoret. Comput. Sci. 454 (2012) $199-205$

20. Aho, A.: Algorithms for Finding Patterns in Strings. In van Leeuwen, J., ed.: Handbook of Theoret. Comput. Sci. Elsevier Science Publishers B. V., Amsterdam, the Netherlands (1990) 257-300

21. Kolpakov, R., Kucherov, G.: Finding Maximal Repetitions in a Word in Linear Time. In: Proceedings of the 40th Annual Symposium on Foundations of Computer Science. FOCS '99, IEEE Computer Society (1999) 596-604

22. Berstel, J.: Sturmian and episturmian words (a survey of some recent results). In Bozapalidis, S., Rohonis, G., eds.: CAI 2007. Volume 4728 of Lecture Notes in Comput. Sci., Springer (2007) 23-47

23. Berstel, J., Lauve, A., Reutenauer, C., Saliola, F.: Combinatorics on Words: Christoffel Words and Repetition in Words. Volume 27 of CRM monograph series. American Mathematical Society (2008)

24. Pytheas Fogg, N.: Substitutions in Dynamics, Arithmetics and Combinatorics. Volume 1794 of Lecture Notes in Math. Springer (2002)

25. Mignosi, F., Pirillo, G.: Repetitions in the Fibonacci infinite word. RAIRO Theor. Inform. Appl. 26 (1992) 199-204

26. Mignosi, F.: Infinite Words with Linear Subword Complexity. Theoret. Comput. Sci. 65 (1989) 221-242

27. Hardy, G.H., Wright, E.M.: An Introduction to the Theory of Numbers. Clarendon Press, Oxford (1979) 5th edition.

28. Mignosi, F.: On the number of factors of Sturmian words. Theoret. Comput. Sci. 82 (1991) 71-84

29. Bucci, M., De Luca, A., Zamboni, L.: Some characterizations of Sturmian words in terms of the lexicographic order. Fundamenta Informaticae 116 (2012) 25-33

30. Glen, A., Justin, J., Pirillo, G.: Characterizations of finite and infinite episturmian words via lexicographic orderings. European Journal of Combinatorics 29 (2008) $45-58$

31. Jenkinson, O., Zamboni, L.Q.: Characterisations of balanced words via orderings. Theoret. Comput. Sci. 310 (2004) 247-271

32. Perrin, D., Restivo, A.: A note on Sturmian words. Theoret. Comput. Sci. 429 (2012) 265-272 